\title{
EFICACIA DE LA CARBOXIMETILCELULOSA SÓDICA PARA EL TRATAMIENTO DEL SÍNDROME DEL OJO SECO
}

\author{
EFFICACY OF SODIUM CARBOXYMETHYLCELLULOSE IN THE \\ TREATMENT OF DRY EYE SYNDROME
}

\author{
BRUIX A ${ }^{1}$, ADÁN A ${ }^{1}$, CASAROLI-MARANO RP ${ }^{1}$
}

\begin{abstract}
RESUMEN
Objetivo: Evaluar la eficacia de la carboximetilcelulosa sódica para el tratamiento del Síndrome de Ojo Seco (SOS).

Material y métodos: Se realizó un ensayo clínico prospectivo randomizado unicéntrico y enmascarado de tipo grupo problema/control con 19 pacientes que padecían un SOS leve o moderado, durante un período de 12 meses. Los pacientes fueron clínicamente evaluados cada 3 meses y tratados con una solución isotónica de carboximetilcelulosa sódica (CMC) al 0,5\% o BSS. La toma de los síntomas subjetivos, las pruebas objetivas de funcionalidad clínica, y la citología de impresión conjuntival fueron realizadas según el protocolo preestablecido. Para la comparación de los datos entre los grupos se utilizó un análisis estadístico mediante prueba de chi cuadrado $\left(\chi^{2}\right)$.

Resultados: Se ha observado una disminución significativa $(p<0,05)$ en la frecuencia de la sintomatología subjetiva asociada a una mejoría significativa $(\mathrm{p}<0,05)$ en la estabilidad de la interfase de la película lagrimal tras el tratamiento con CMC. Hubo una tendencia a la mejoría del grado de humecta-
\end{abstract}

\begin{abstract}
Aim: To assess the efficacy of sodium carboxymethylcellulose in the treatment of dry eye.

Material and methods: We carried out a prospective, randomized, masked-observer, control/problem group, single-center clinical assay during a period of 12 months in 19 patients that presented mild or moderate forms of dry eye. Patients were clinically evaluated each 3 months and treated with a $0.5 \%$ isotonic solution of sodium carboxymethylcellulose (CMC) or balanced salt solution. Subjective symptoms, functional tests and conjunctival impression cytology were performed according preexistent schedule study visits. To compare data between groups chi squared $\left(\chi^{2}\right)$ analysis was applied. Results: We observed a significant $(\mathrm{p}<0.05)$ decrease in the frequency of subjective symptoms and a significant $(\mathrm{p}<0.05)$ improvement of tearfilm interface stability after CMC treatment. There was a tendency to improve the degree of corneal surface wettability and the tearfilm integrity with higher percentage improvements in the CMC group compared to controls. Improved baseline values in at least one of the objective functional tests carried out $(\mathrm{p}<0.05)$
\end{abstract}

Recibido: 11/5/05. Aceptado: 20/2/06.

Servicio de Oftalmología. Hospital de la Santa Creu i Sant Pau, Facultad de Medicina de la Universidad Autónoma de Barcelona. España.

1 Doctor en Medicina.

Comunicación presentada parcialmente en el LXXIX Congreso de la S.E.O. (Valencia 2003).

Correspondencia:

Ricardo P. Casaroli-Marano

Departamento de Biología Celular

Universidad de Barcelona

Avda. Diagonal 645

08028 Barcelona

España

E-mail: rcasaroli@ub.edu 
ción de la superficie corneal y de la integridad de la película lagrimal, con un porcentaje superior de mejorías en el grupo problema. Se constató que un mayor porcentaje de pacientes en el grupo problema $(83,3 \%)$ con relación al grupo control (34\%), mejoraron por lo menos en una de las pruebas funcionales de evaluación $(\mathrm{p}<0,05)$. Asimismo, se observó una tendencia a la disminución de la frecuencia de síntomas subjetivos concomitantes tras el tratamiento con CMC. La citología de impresión (CI) no ha permitido establecer diferencias significativas con relación a la respuesta clínica al tratamiento.

Conclusiones: Se pudo constatar un efecto significativamente favorable de la CMC en la mejoría de los parámetros clínicos del SOS leve y moderado.

Palabras clave: lágrimas artificiales, carmelosa sódica, queratoconjuntivitis sicca, sustitutos de la lágrima, síndrome de Sjögren. was also observed in an elevated percentage of patients in the CMC group (83.3\%) compared with controls (34\%). Furthermore, we observed a tendency to diminish the frequency of associated subjective symptoms after treatment. Conjunctival impression cytology did not provide significant differences related with therapeutic response.

Conclusions: The results show a significant beneficial effect of CMC to improve clinical parameters in mild and moderate forms of dry eye (Arch Soc Esp Oftalmol 2006; 81: 85-92).

Key words: Artificial tears, sodium carmellose, keratoconjunctivitis sicca, tear substitutes, Sjögren's syndrome.

\section{INTRODUCCIÓN}

El Síndrome del Ojo Seco (SOS) está caracterizado por un conjunto de alteraciones de la superficie ocular que pueden relacionarse con la calidad de la lágrima, la composición normal de la película lagrimal, y alteraciones en el parpadeo o en el cierre regular de los párpados $(1,2)$, que conlleva una reducción de la estabilidad de la película lagrimal y la alteración de la superficie ocular $(3,4)$.

En individuos mayores de 45 años, alrededor de $20 \%$ de las mujeres y $15 \%$ de los hombres presentan síntomas de sequedad ocular que, clínicamente, se presentan con diferentes grados de severidad (1,5-7). El ojo seco severo es el menos frecuente y el de más difícil manejo, mientras que el ojo seco leve y moderado son los que más se presentan en la práctica diaria (1-4).

Recientes estudios (7-9) evidencian qué alteraciones de tipo inmunológicas están relacionadas con la patogénesis del SOS, no solamente en el Síndrome de Sjögren, sino también en las alteraciones post-infecciosas y en el envejecimiento. No obstante, en la actualidad, existen bases suficientes para considerar que una irritación crónica de la superficie ocular puede actuar como desencadenante en una superficie ocular predispuesta por factores hormonales o inmunológicos (10).
El tratamiento del SOS plantea algunas dificultades por corresponder a una alteración crónica que implica una terapia de tipo prolongado y con cumplimiento estricto $(2,11)$. Por lo tanto, un hecho importante para el éxito terapéutico en el SOS es la orientación médica y la comprensión de la finalidad de las medidas terapéuticas por parte del paciente.

El objetivo de presente trabajo fue el de evaluar la eficacia de una solución de carboximetilcelulosa sódica (CMC-carmelosa sódica) como sustituto de la lágrima para el tratamiento del SOS. Asimismo, se valoró la presencia y la severidad de los síntomas y signos de ojo seco mediante la utilización: a) de síntomas subjetivos como parámetro de utilidad para el diagnóstico clínico y el seguimiento de la respuesta terapéutica, b) de la exploración básica mediante pruebas de funcionalidad para la evaluación del ojo seco y c) de la citología de impresión conjuntival (CI) como criterio para el seguimiento clínico de la patología frente a la respuesta terapéutica.

\section{SUJETOS, MATERIAL Y MÉTODOS}

\section{Diseño y población de estudio}

El estudio se proyectó como un ensayo clínico unicéntrico, prospectivo temporal y alecitorizado, 
de tipo enmascarado con grupo problema/control y duración de 12 meses. El ensayo fue conducido de acuerdo con los principios éticos para la investigación con seres humanos (12). Cada paciente fue informado detalladamente del objetivo y propósitos del estudio y se obtuvo el consentimiento informado para su participación. Todos los pacientes fueron inicialmente evaluados por un único oftalmólogo y clasificados según los grados de severidad del SOS: a) Leve; cuando cumplían 1 ó 2 criterios, b) Moderado; cuando cumplían 3 ó 4 criterios, c) Severo; cuando cumplían más de 4 criterios.

Los pacientes con cuadro clínico severo no fueron incluidos en el ensayo. También se excluyeron los que presentaban patologías inflamatorias de la superficie ocular o en el segmento anterior, o habían sido diagnosticados de glaucoma. El uso de lentes de contacto y/o la utilización de medicación tópica o sistémica que pudiesen interferir en la producción de la lágrima (2), también fueron considerados criterios de exclusión. Asimismo se excluyeron los pacientes sometidos a cirugía ocular o con episodio de trauma ocular en el año anterior al inicio del estudio.

Para la elección de los grupos de tratamiento (CMC) y control (BSS) se utilizó un protocolo de inclusión del tipo alecitorizado, en el cual los pacientes involucrados en el ensayo fueron asignados a una razón de 2:1 (tratamiento:control) según su orden de entrada. La asignación en el grupo de afectación, la pauta terapéutica, y las evaluaciones clínicas hasta la finalización del estudio, fueron determinadas y realizadas por los oftalmólogos participantes en el ensayo según cronograma y protocolo preestablecido (tabla I).

Tabla I. Protocolo de evaluación clínica y esquema de seguimiento del estudio

\begin{tabular}{lcccc}
\hline Actividad & día & mes & mes & mes \\
& 0 & 3 & 6 & 12 \\
\hline Consentimiento informado & $*$ & & & \\
Evaluación oftalmológica general & $*$ & $*$ & $*$ & $*$ \\
Anamnésis médica y oftalmológica & $*$ & & & \\
Randomización & $*$ & & & \\
Asignación del tratamiento & $*$ & & & $*$ \\
Pruebas funcionales objetivas & $*$ & & & $*$ \\
Síntomas subjetivos & $*$ & $*$ & $*$ & $*$ \\
Cuestionario matizado de síntomas & $*$ & & & $*$ \\
Citología de Impresión & $*$ & & & $*$ \\
Tratamiento & $*$ & $*$ & $*$ & $*$ \\
Evaluación de la eficácia & $*$ & $*$ & $*$ & $*$ \\
Evaluación de efectos adversos & & $*$ & $*$ & $*$ \\
\hline \hline
\end{tabular}

\section{Criterios de evaluación clínica}

Los pacientes fueron evaluados cada 3 meses en los primeros 6 meses, y a los 12 meses se realizó una evaluación clínica oftalmológica final (tabla I).

Las pruebas funcionales de evaluación objetivas para el SOS, fueron realizadas en la primera (día 0) y en la última (mes 12) evaluación, según las técnicas y protocolos descritos (4). Dichas pruebas, fueron aplicadas en un orden recomendado (4) con el objetivo de mitigar la interferencia de una prueba sobre la siguiente.

Asimismo, se empleó un cuestionario diagnóstico matizado de contestación sencilla (13), modificado para este estudio, que se facilitó a los pacientes en la primera y en la última evaluación clínica del estudio.

\section{Material del estudio}

Para el grupo problema, se administró una solución isotónica de carboximetilcelulosa sódica al $0,5 \%$ sin conservantes ya comercializada (Cellufresh $^{\circledR}$, Allergan SA, Madrid), que fue comparada con la aplicación de Solución Salina Balanceada (BSS, Alcon-Cusi SA, Barcelona) en el grupo control. Ambos productos fueron suministrados en su presentación original y se recomendó a los pacientes la utilización tópica ocular de 1 a 2 gotas del producto de 3 a 4 veces al día como mínimo, o según la necesidad. No se permitió la utilización de otro tipo de medicación tópica ocular durante la realización del ensayo.

\section{Citología de Impresión Conjuntival}

La citología de impresión (CI) se realizó de acuerdo a las técnicas descritas con anterioridad (14). Se utilizó el criterio de Nelson (15) para la interpretación y para establecer la clasificación del grado de afectación conjuntival. Las citologías fueron realizadas antes y después del tratamiento con un periodo mínimo de 6 meses y máximo de 1 año entre las evaluaciones. La preparación, tinción y la interpretación de los hallazgos en los especimenes fueron realizadas por un único citopatólogo. 


\section{Tratamiento estadístico}

La recogida y análisis de los datos obtenidos de los resultados se registraron en un programa de hoja de cálculo (Microsolf Excel, Office Windows 2000). Para la comparación entre grupo proble$\mathrm{ma} / \mathrm{control}$ se utilizó un análisis estadístico mediante prueba de chi cuadrado $\left(\chi^{2}\right)$ cuando los datos así lo permitieron.

\section{RESULTADOS}

Fueron examinados 45 pacientes de entre los cuales solamente 19 pacientes cumplieron los criterios de inclusión y las evaluaciones determinadas previamente en el protocolo del ensayo. Según los criterios de evaluación clínica, el $11 \%$ de los pacientes $(n=5)$ tenían un SOS con grado de afectación severa y, por ello, recibieron tratamiento inmediato siendo excluidos del estudio. Así, el grupo problema quedó constituido por 13 pacientes y el grupo control por seis pacientes, todos ellos de sexo femenino. La media de edad del grupo problema fue de 56,8 años (entre 42 y 72 años) y para el grupo control de 62 años (entre 52 y 72 años). En los grupos problema y control el $84,5 \%(n=11)$ y el $33,4 \%(n=2)$ respectivamente presentaban SOS con grado de afectación moderada. El periodo medio de seguimiento clínico fue de 9 meses y medio, variando entre 6 y 12 meses.

Se registró una alta frecuencia de patologías sistémicas asociadas en ambos grupos del estudio. El síndrome de Sjögren (33\%-38\%) y las poliartralgias (30\%-33\%) fueron las asociaciones más frecuentemente observadas. Solamente un $23 \%$ de los pacientes del grupo problema y un $33 \%$ de los pacientes del grupo control no presentaban patologías asociadas al SOS.

\section{Sintomatología subjetiva}

Con el objetivo de evaluar la sintomatología antes y después del tratamiento, se elaboró un cuestionario matizado con doce síntomas de tipo subjetivo que los pacientes contestaron en la visita inicial y al finalizar el estudio. Se observó una disminución significativa $(p<0,05)$ en la frecuencia de algunos de los síntomas subjetivos tras el tratamiento con CMC. En el grupo control, no se constató variaciones en la frecuencia de los síntomas.
Con el objetivo de observar la concomitancia de dichos síntomas, se clasificaron los pacientes según el número de síntomas referidos en los siguientes grupos: a) con hasta 3 síntomas, b) con hasta 6 síntomas, c) con hasta 9 síntomas, d) con más de 9 síntomas y e) con todos los síntomas. Se pudo constatar que el 61,5\% de los pacientes $(n=8)$ en el grupo problema presentaban entre 7 y 9 síntomas concomitantes antes del tratamiento, reduciéndose al $46 \%(\mathrm{n}=6)$ después del tratamiento. En el mismo grupo, el $23 \%$ de los pacientes $(n=3)$ presentaron como máximo hasta 3 síntomas al final del estudio. Asimismo, se constató después del tratamiento con CMC un desplazamiento del número de pacientes hacia los grupos que presentaban menor número de síntomas concomitantes (fig. 1). No hubo variaciones de frecuencia en el grupo control tratado con BSS.

Durante el estudio no se constató la aparición de efectos adversos como consecuencia de la utilización de los productos. Ambos tratamientos fueron bien tolerados sin compromiso para la agudeza visual de los participantes con la excepción de discreta borrosidad visual inmediatamente después de la instilación.

\section{Pruebas funcionales objetivas}

Con el fin de evaluar la mejoría clínica, se utilizaron pruebas funcionales de evaluación objetivas para la valoración del SOS (ver materiales y métodos).

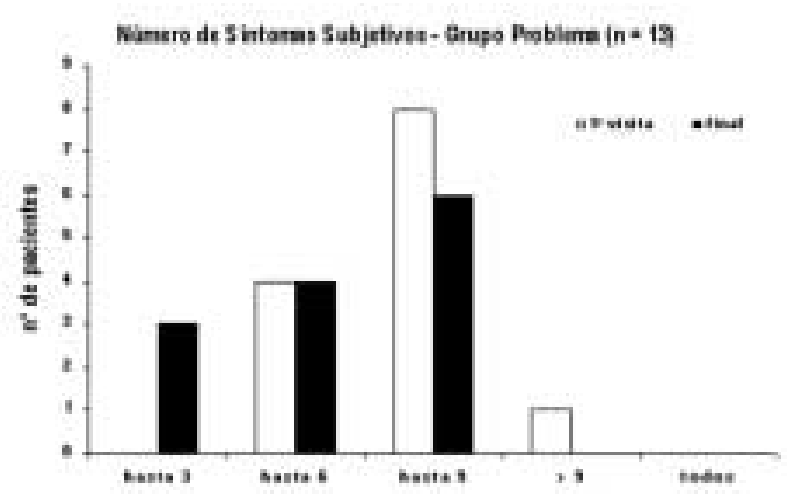

Fig. 1: Frecuencia de síntomas subjetivos antes (barras blancas) y después (barras negras) del tratamiento con CMC en los pacientes del grupo problema $(n=13)$. Se observó una tendencia a la disminución de síntomas concomitantes tras el tratamiento. 
La prueba de Schirmer basal, realizada tras la instilación de colirio anestésico, evidenció una tendencia a la mejoría del grado de humectación en los ojos del grupo problema. No obstante, las diferencias observadas no fueron estadísticamente significativas. Asimismo, se registró un porcentaje superior de ojos que mejoraron su grado de humectación en el grupo problema $(34,8 \%)$ con relación al control $(25 \%)$. Además, se constató un mayor porcentaje de empeoramiento del grado de humectación en ojos del grupo control (25\%) con relación al problema $(8,7 \%)$.

La integridad del epitelio de la córnea fue estudiada mediante la instilación de gotas de fluoresceína sódica al $2 \%$ y semi-cuantificada según el grado de afectación entre 0 y 3 cruces. Solamente el $23 \%$ de los pacientes $(n=3)$ del grupo problema y el $16,7 \%(n=1)$ del grupo control, presentaban alteraciones de la superficie corneal. Después del tratamiento con CMC, todos los pacientes del grupo problema han experimentado mejoría en el grado de afectación del epitelio corneal, mientras que no se observó mejoría en el paciente del grupo control.

La tinción con Rosa de Bengala evidenció una tendencia a la mejoría de la integridad de la película lagrimal en los pacientes del grupo problema. Asimismo, se constató un mayor porcentaje de ojos que mejoraron la integridad de su película lagrimal en el grupo problema (50\%) cuando se comparó con el grupo control $(33,3 \%)$, sin que esta diferencia fuera estadísticamente significativa. A pesar de que la mitad de los ojos examinados en el grupo problema hayan experimentado mejoría, el 41,7\% de los ojos restantes con tinción positiva, no presentaron cambios en la valoración. En el grupo control, cuatro ojos $(33,4 \%)$ presentaban tinción negativa para la prueba.

El tiempo de ruptura de la película lagrimal (BUT), que se valoró como una medida de la estabilidad de la interfase entre la película lagrimal y la superficie ocular, mostró una mejoría significativa $(p<0,05)$ a favor del grupo problema. Se ha observado un porcentaje superior de ojos que mejoraron el BUT en el grupo problema (50\%) con relación al grupo control $(16,6 \%)$. Es de resaltar que el $21 \%$ de ojos del grupo problema y el $33 \%$ de ojos del grupo control presentaban BUT compatibles con la normalidad antes del tratamiento (fig. 2).

La evaluación de la integridad del menisco lagrimal no ha permitido establecer diferencias de respuesta entre los grupos. Todos los ojos estudiados

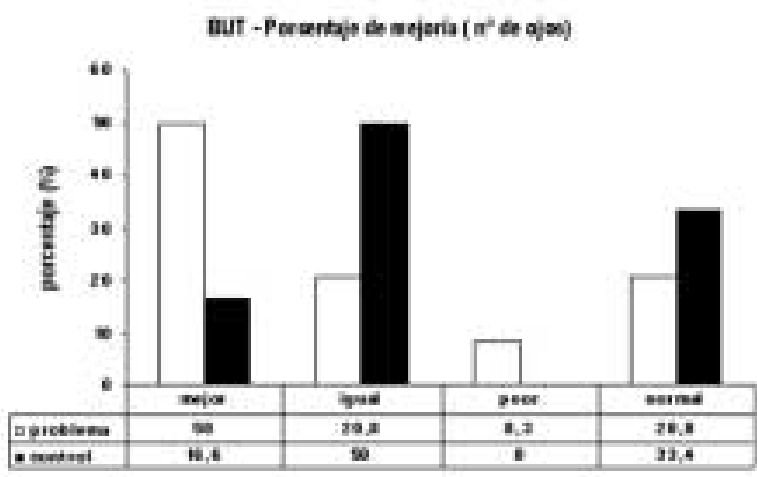

Fig. 2: Mejoría del tiempo de ruptura de la película lagrimal (BUT) tras el tratamiento. Se pudo observar un mayor porcentaje de mejoría $(50 \%)$ en el grupo problema (barras blancas), tratado con CMC, con relación al grupo control (16,6\%) (barras negras) tratado con BSS. Esta diferencia fue significativa $(p<0,05)$.

en el grupo control y el $64 \%$ de los ojos del grupo problemas, no presentaban alteraciones de la integridad del menisco lagrimal antes del tratamiento.

Finalmente, hemos intentado establecer una evaluación funcional globalizada de la respuesta terapéutica teniendo en consideración los resultados observados en las pruebas objetivas más indicativas en el presente estudio: a) prueba de Schirmer, b) tinción con fluoresceína sódica, c) tinción con Rosa de Bengala, d) BUT. Se estableció una puntuación para la cuantificación de una mejoría (+ 2 puntos), una igualdad (+ 1 punto) y un empeoramiento (cero puntos) con relación al resultado de la prueba. Así, una puntuación (score) mayor o igual ( $\geq$ ) a 5 puntos representa la mejora de por lo menos una de las pruebas de evaluación objetiva. Con dicho criterio, se constató que el $83,3 \%$ de los pacientes $(\mathrm{n}=10)$ del grupo problema y el $34 \%(n=2)$ del grupo control, presentaban una puntuación $\geq 5$ puntos al considerarse los resultados obtenidos en las pruebas de funcionalidad objetivas al final del estudio (fig. 3). Esta diferencia fue significativa $(\mathrm{p}<0,05)$.

\section{Citología de impresión conjuntival}

Se ha realizado CI en 14 pacientes -24 ojos del grupo problema y cuatro ojos del grupo controlantes y después del tratamiento con el objetivo de verificar la eficacia terapéutica en la evolución del 


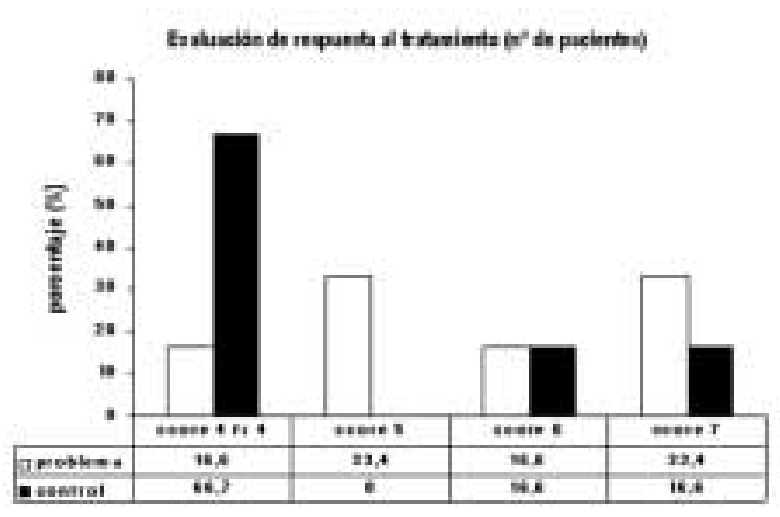

Fig. 3: Figura 3: Valoración funcional global de la respuesta terapéutica, según la puntuación (mejoría $=2$; igualdad $=1$; empeoramiento $=0$ ) establecida para las pruebas de evaluación objetiva en el grupo problema (barras blancas), tratado con CMC, y el grupo control (barras negras) tratado con BSS. Las puntuaciones (score) $\geq 5$ representan la mejoría de por lo menos una de las pruebas funcionales de evaluación objetiva tras el tratamiento. Se pudo observar un mayor número de pacientes con scores $\geq 5$ en el grupo problema $(83,3 \%)$ en relación al grupo control (34\%). Esta diferencia fue significativa $(p<0,05)$.

SOS, así como también la aplicabilidad de la prueba para el control del seguimiento clínico. Los especimenes fueron obtenidos preferentemente de la conjuntival bulbar inferior. Los criterios de clasificación citopatológica fueron los anteriormente descritos (15).

Antes del tratamiento con CMC, los ojos en el grupo problema se distribuyeron en: $70,8 \%(n=17)$ en el grado $1,16,6 \%(n=4)$ en el grado 2 , con $12,5 \%(n=3)$ de resultados normales. Tras el cumplimiento del tratamiento se observó una ligera disminución del porcentaje de ojos clasificados en el grado $1(62,5 \%, n=15)$ con el consecuente incremento en el grupo correspondiente al grado $2(25 \%$, $\mathrm{n}=6$ ). Esta diferencia no fue estadísticamente significativa. En el grupo control, al inicio del estudio, 3 ojos fueron clasificados en grado 1 y un examen tuvo resultado normal. Al final del ensayo, todos los ojos del grupo control, tratados con BSS, se clasificaron como grado 1.

No se observaron diferencias significativas con relación al grado de afectación de las células caliciformes y los estados metaplásicos de la conjuntiva entre los grupos del ensayo antes y después del tratamiento. Asimismo, la CI no se correlacionó con la mejoría de la sintomatología subjetiva observada o la mejoría de algunos de los parámetros de los ensayos funcionales de evaluación objetiva. Sin embargo, se constató en la CI inicial una mayor prevalencia $(87,5 \%)$ de afectación de las células caliciformes en ojos del grupo problema.

\section{DISCUSIÓN}

El presente estudio constata que la instilación frecuente, continuada y a largo plazo de una solución isotónica de CMC tiene efecto beneficioso para los cuadros leves y moderados de ojo seco comparados con la utilización control de BSS. Se ha podido comprobar una mejoría significativa de la sintomatología subjetiva asociada a la patología, además del incremento de los valores de base observados en algunas pruebas funcionales objetivas realizadas para la valoración de la afectación de la superficie ocular. Dichos resultados son compatibles y corroboran trabajos anteriores sobre la eficacia y la tolerancia de los sustitutos de la lágrima que contienen CMC en solución (16-18).

El SOS es una patología frecuente en la clínica diaria y la mayor parte de los abordajes terapéuticos para dicha enfermedad tienen esencialmente como punto común la utilización de sustitutos de la lágrima sin conservantes $(2,7,10)$. Se asume que un sustituto de la lágrima ideal para el tratamiento del SOS debe presentar algunas características esenciales, tales como, la ausencia de toxicidad, una viscosidad adecuada, no interferir en la agudeza y la capacidad visual, no alterar la producción de mucina, poseer función humectante, además de colaborar en la estabilización de la película lagrimal y presentar un elevado tiempo de permanencia sobre la superficie ocular $(2,11)$.

La hiperosmolaridad lagrimal ha sido considerada uno de los mecanismos primarios que desencadena la sintomatología subjetiva de desconfort y el proceso inflamatorio asociado que se observa en la superficie ocular (19). Asimismo, estudios de la fisiología de la película lagrimal demostraron que la presencia de algunos elementos, tales como el bicarbonato y potasio en la lágrima, son importantes no solo para su equilibrio electrolítico sino también para la integridad de la superficie ocular $(2,20,21)$. La solución de CMC utilizada en el presente estudio presenta una composición electrolítica similar a la de la lágrima humana y, por el hecho de ser isotónica, seguramente puede contribuir a la 
corrección de las alteraciones de la osmolaridad y el $\mathrm{pH}$ que generalmente se observan en el SOS. Creemos que todo ello podría explicar la disminución significativa observada en la frecuencia de la sintomatología subjetiva en el grupo problema. Además, podemos asociar dicha mejoría al incremento de la humectación de la superficie corneal tal como se detectó en los valores obtenidos con la prueba de Schirmer.

Las células caliciformes situadas en la superficie ocular (22) son las productoras de la capa de mucina, esencial para la estabilidad de la película lagrimal (2). Investigaciones previas (21) sugirieron que las soluciones con CMC tienen la capacidad de restituir la funcionalidad de las células caliciformes y mejorar los estados metaplásicos previos de las células epiteliales de la conjuntiva observados mediante CI (16). Desafortunadamente en nuestro estudio, la CI no pudo demostrar diferencias respecto al grado de afectación de las células caliciformes en ambos grupos antes y después del tratamiento. No obstante, se observó una mejoría significativa de la estabilidad de la interfase entre la película lagrimal y la superficie ocular en el grupo problema, mediante la valoración del BUT y la prueba del Rosa de Bengala.

De entre las propiedades de la CMC se encuentra su característica viscoelástica (11) que contribuye en la lubricación de la superficie ocular. Este hecho, aumenta la estabilidad de la película lagrimal precorneal que, a su vez, protege la superficie ocular contra las agresiones ambientales (8). Asimismo, por sus características moleculares de polisacárido (11), la CMC presenta propiedad retensiva que favorece la permanencia del compuesto e incrementa la humectación de la superficie ocular (23). Así, todo ello podría explicar el mayor porcentaje de pacientes $(83,3 \%)$ en el grupo problema que presentó mejoría significativa en por lo menos una de las pruebas de evaluación objetiva al finalizar el estudio, con relación al grupo control (34\%) al que se administró BSS.

Por otro lado, ya se conoce que la ausencia de conservantes en los sustitutos de la lágrima es esencial para evitar efectos tóxicos sobre el epitelio corneal y no empeorar el proceso inflamatorio asociado al $\operatorname{SOS}(2,7,10,11)$.

En nuestro estudio es importante resaltar la elevada frecuencia de patologías sistémicas asociadas al SOS. El Síndrome de Sjögren y las poliartralgias fueron las más encontradas. Se sabe que muchas de las enfermedades vasculares que afectan el colágeno, de entre las cuales se incluyen varias patologías autoinmunes, suelen presentarse asociadas con síntomas de ojo seco en diferentes grados de severidad (10). Se considera que la reducción de la secreción lagrimal, presente en dichas patologías, es una consecuencia de la infiltración de células inflamatorias en las glándulas lagrimales que pueden o no igualmente afectar la conjuntiva $(9,24)$.

En el presente ensayo, como consecuencia de la alecitorización empleada para la elección del tratamiento, hay una mayor frecuencia de pacientes con un grado de afectación moderada en el grupo problema $(84,5 \%)$ con relación al grupo control $(33,4 \%)$. Aunque el empleo aislado de tratamiento tópico con gotas representó la mejoría del cuadro clínico en una parte significativa de los pacientes estudiados, una posible asociación terapéutica complementaria, muy probablemente, elevaría el porcentaje de beneficios observados. Cabe recordar que en los casos de afectación severa, en los cuales se observan la queratinización conjuntival, daño corneal en diferentes grados, presencia de filamentos y defectos epiteliales graves, son recomendadas otras estrategias terapéuticas coadyuvantes $(2,7,10,11)$.

Finalmente, la seguridad y la eficacia de la CMC fueron evaluadas de acuerdo a la tolerancia, a la aparición de posible efectos adversos y a las medidas registradas en las pruebas objetivas realizadas para la evaluación del estado de la superficie ocular. La ausencia de sintomatología adversa y los resultados observados sobre la tolerancia, permite concluir que la aplicación frecuente de una solución isotónica de CMC al 0,5\% sin conservantes en la superficie ocular es segura, bien tolerada y capaz de contribuir en el alivio de la sintomatología subjetiva en los casos de SOS de afectación leve y moderada. A nuestro parecer el hecho de que el sustituto de la lágrima elegido tenga una respuesta positiva sobre la sintomatología subjetiva, y a pesar de las otras consideraciones, es uno de los parámetros importantes y seguramente el que contribuye a un mejor cumplimiento del tratamiento a largo plazo.

\section{AGRADECIMIENTOS}

A la Dra. Elena Sagristà, de la Universidad de Barcelona, por su lectura crítica del manuscrito. 


\section{BIBLIOGRAFÍA}

1. Brewitt H, Sistani F. Dry eye disease: the scale of the problem. Surv Ophthalmol 2001; 45: S199-S202.

2. Johnson ME, Murphy PJ. Changes in the tear film and ocular surface from dry eye syndrome. Prog Retin Eye Res 2004; 23: 449-474.

3. Holly FJ, Lemp MA. Tear physiology and dry eyes. Surv Ophthalmol 1977; 22: 69-87.

4. Bron AJ. Diagnosis of dry eye. Surv Ophthalmol 2001; 45: S221-S226.

5. Caffery BE, Richter D, Simpson T, Fonn D, Doughty M, Gordon K. CANDEES. The Canadian Dry Eye Epidemiology Study. Adv Exp Med Biol 1998; 438: 805-806.

6. Hikichi T, Yoshida A, Fukui Y, Hamano T, Ri M, Araki K, et al. Prevalence of dry eye in Japanese eye centers. Graefes Arch Clin Exp Ophthalmol 1995; 233: 555-558.

7. Perry HD, Donnenfeld ED. Dry eye diagnosis and management in 2004. Curr Opin Ophthalmol 2004; 15: 299304.

8. Stern ME, Beuerman RW, Fox RI, Gao J, Mircheff AK, Pflugfelder SC. The pathology of dry eye: the interaction between the ocular surface and lacrimal glands. Cornea 1998; 17: 584-589.

9. Baudouin C. The pathology of dry eye. Surv Ophthalmol 2001; 45: S211-S220.

10. Smith RE. The tear film complex: pathogenesis and emerging therapies for dry eyes. Cornea 2005; 24: 1-7.

11. Calonge M. The treatment of dry eye. Surv Ophthalmol 2001; 45: S227-S239.

12. Medical Research Council (MRC). Guidelines for good clinical practice in clinical trials. London: MRC; 1998.

13. McMonnies CW. Key questions in a dry eye history. J Am Optom Assoc 1986; 57: 512-517.
14. Pflugfelder SC. Advances in the diagnosis and management of keratoconjunctivitis sicca. Curr Opin Ophthalmol 1998; 9: 50-53.

15. Nelson JD, Havener VR, Cameron JD. Cellulose acetate impressions of the ocular surface. Dry eye states. Arch Ophthalmol 1983; 101: 1869-1872.

16. Grene RB, Lankston P, Mordaunt J, Harrold M, Gwon A, Jones $R$. Unpreserved carboxymethylcellulose artificial tears evaluated in patients with keratoconjunctivitis sicca. Cornea 1992; 11: 294-301.

17. Diebold Y, Herreras JM, Callejo S, Argueso P, Calonge M. Carbomer-versus cellulose-based artificial-tear formulations: morphologic and toxicologic effects on a corneal cell line. Cornea 1998; 17: 433-440.

18. Lenton LM, Albietz JM. Effect of carmellose-based artificial tears on the ocular surface in eyes after in situ keratomileusis. J Refract Surg 1999; 15: S227-S231.

19. Lemp MA. Report of the National Eye Institut / Industry workshop on Clinical Trials in Dry Eyes. CLAO J 1995; 21: $221-232$.

20. Lopez Bernal D, Ubels JL. Artificial tear composition and promotion of recovery of the damaged corneal epithelium. Cornea 1993; 12: 115-120.

21. Gilbard JP, Rossi SR. An electrolyte-based solution that increases corneal glycogen and conjunctival goblet-cell density in a rabbit model for keratoconjunctivitis sicca. Ophthalmology 1992; 99: 600-604.

22. Dartt DA. Control of mucin production by ocular surface epithelial cells. Exp Eye Res 2004; 78: 173-185.

23. Snibson GR, Greaves JL, Soper ND, Tiffany JM, Wilson $C G$, Bron AJ. Ocular surface residence times of artificial tear solutions. Cornea 1992; 11: 288-293.

24. Jabs DA. The eye and rheumatic disease. Bull Rheum Dis 1998; 47: 5-10. 